\title{
Development and validation of the Japanese version of the Gambling Related Cognitions Scale (GRCS-J)
}

Kengo Yokomitsu ${ }^{1 *}$, Takahito Takahashi ${ }^{2}$, Junichiro Kanazawa ${ }^{3}$ and Yuji Sakano ${ }^{3}$

\author{
* Correspondence: \\ yokomitsuken5@gmail.com \\ ${ }^{1}$ Tobacco Academic Studies Center, \\ Tokyo, Japan \\ Full list of author information is \\ available at the end of the article
}

\begin{abstract}
This study aimed to develop and test the Japanese version of the Gambling Related Cognitions Scale (GRCS-J) to investigate its reliability and validity for assessing gambling cognitions in a Japanese sample. Five hundred and thirty-six participants (351 male, 185 female; Mean age = 29.75 years) from a community sample living in Japan were included in the analyses. The results of a confirmatory factor analysis confirmed that a five-factor model was appropriate for the data (goodness of fit index $=.87$, comparative fit index $=.90$, root mean square error of approximation $=.07$ ). The Cronbach's alpha coefficient was .94 for the total scale and ranged from .74 to .85 for the subscales. The concurrent validities for the GRCS-J were also good. Further, the results of a t-test revealed significant gender differences in the GRCS-J subscale scores and the total score. These results indicated that the GRCS-J was a valid and reliable measure for assessing gambling cognitions in a non-clinical Japanese sample.
\end{abstract}

\section{Background}

The life-time prevalence of pathological gambling in people who speak English and other European languages has been found to be between 0.8 and 1.2\% (Stucki and Rihs-Middel 2007). In Japan, the epidemiological research on pathological gambling has never been conducted. Sato (2008) reported that many pathological gamblers in Japan did not receive medical care or counseling and that pathological gamblers and their families faced gambling-related problems such as financial, legal, and occupational problems. Treatment facilities for pathological gamblers in Japan have also been found to be insufficient (Moriyama 2008), and therefore a large number of these gamblers are unable to receive treatment.

Gambling research has highlighted the role of gambling-related cognitions in the development and maintenance of gambling habits (e.g., Blaszczynski and Nower 2002). For example, $70 \%$ of gambler's reported verbalizations were erroneous when using the thinking aloud method while gambling (Gaboury and Ladouceur 1989). Moreover, studies on the habits of heavy gamblers revealed that while playing roulette, lotteries, or blackjack, gamblers were more irrational when predicting outcomes than non-gamblers (Toneatto et al. 1997). Therefore, it could be concluded that gamblers are more likely to make irrational choices when attempting to predict outcomes based on chance events (Petry 2005). Other research identified that gambling-related cognitions were significantly related to 
gambling severity (Hedge's $g$ statistic: 0.77 to 2.50 ) (Goodie and Fortune 2013), and some reviews showed severe gambling symptoms could be associated with other comorbidities (Lorains et al. 2011).

Cognitive behavioral therapy is regarded as the most effective treatment for pathological gambling (Toneatto and Ladouceur 2003) as it assists gamblers to reduce their gambling behavior (Cowlishaw et al. 2012), become aware of the irrational cognitions which triggers their gambling desire, and to learn to correct it (Fortune and Goodie 2012). For example, Ladouceur and colleagues found that pathological gamblers who acquired the skills in treatment to identify and correct their gambling-related cognitions were able to effectively reduce their gambling behavior (Ladouceur et al. 2001). Thus, there have been many clinical studies focusing on gambling-related cognitions in Western countries. In Japan, however, there is a lack of clinical and observational studies for pathological gambling. Given the link between gambling-related cognitions and pathological gambling, the availability of a Japanese validated self-report questionnaire for assessing gambling-related cognitions is a necessary preliminary step toward any research on gambling-related cognitions and on the efficacy of cognitive behavioral therapy for pathological gamblers.

A number of scales for measuring gambling-related cognitions have been developed. Of these, the Gambling Related Cognitions Scale (GRCS; Raylu and Oei 2004) has been found to be the most reliable (the overall scale: Cronbach's $\alpha=.93$, the factors: Cronbach's $\alpha=.77-.91$ ) and most valid (the overall scale for the correlation coefficient with the South Oaks Gambling Screen (Lesieur and Blume 1987): $r=.43$ ) tool for assessing gambling-related cognitions (Whelan et al. 2007). Further, a GRCS was developed for Chinese (Oei et al. 2007) and French samples (Grall-Bronnec et al. 2012).

Given that the recognition of gambling-related cognitions is crucial for the moderation of gambling behavior and pathological gambling (Blaszczynski and Nower 2002), it is important to develop and validate a self-report measure to assist researchers and clinicians assess gambling-related cognitions. So, developing a Japanese version of the GRCS (GRCS-J) could be useful for verifying the treatment effects of cognitive behavioral therapy for pathological gamblers, and thus could be used for future gambling treatments in Japan. Therefore, the purpose of the current study is to develop a GRCS-J and examine its validity and reliability in a community sample in Japan.

\section{Method}

Participants

A paper questionnaire was delivered to 1,791 Japanese individuals, aged 18 or older from September 15, 2009 to November 30, 2009. Participants were asked to participate in the study regardless of their gambling frequency. Participants were recruited in several ways. Questionnaires were distributed to university Japanese students in Kagoshima, Kyoto, and Sapporo and sent to Japanese workers in Fukuoka, Osaka, Kyoto, Aichi, Tokyo, and Sapporo. Out of the 852 (47.5\%) responses received, 316 were deemed invalid. Thus, $536(29.9 \%)$ properly completed questionnaires were used for data analyses in this study. Of these 536 people, $65.5 \%(n=351)$ were male and $34.5 \%(n=185)$ were female. The mean age of the participants was 29.75 years $(S D=11.84$; range $=18-87)$. Table 1 outlines the participant demographics. 
Table 1 Demographic characteristics

\begin{tabular}{|c|c|c|c|c|}
\hline & $\begin{array}{l}\text { Total } \\
\mathrm{n}=536 \\
\%\end{array}$ & $\begin{array}{l}\text { NPG } \\
n=248 \\
\%\end{array}$ & $\begin{array}{l}\text { AG } \\
n=195 \\
\%\end{array}$ & $\begin{array}{l}\text { PG } \\
n=93 \\
\%\end{array}$ \\
\hline \multicolumn{5}{|l|}{ Sex } \\
\hline Male & 65.5 & 51.6 & 73.3 & 86.0 \\
\hline Female & 34.5 & 48.4 & 26.7 & 14.0 \\
\hline \multicolumn{5}{|l|}{ Age } \\
\hline $18-19$ years & 9.3 & 12.1 & 8.2 & 6.5 \\
\hline 20-29 years & 55.6 & 55.6 & 55.4 & 53.7 \\
\hline 30-39 years & 16.1 & 17.0 & 15.4 & 15.1 \\
\hline 40-49 years & 9.1 & 6.0 & 11.3 & 12.9 \\
\hline $50-59$ years & 7.3 & 6.5 & 8.2 & 7.5 \\
\hline$\geqq 60$ years & 2.6 & 2.8 & 1.5 & 4.3 \\
\hline \multicolumn{5}{|l|}{ Education level } \\
\hline Elementary and junior high school & 1.1 & 0.8 & 0.5 & 3.2 \\
\hline High school (equivalent test) & 10.5 & 8.1 & 12.8 & 12.9 \\
\hline Junior and career college & 8.9 & 10.1 & 10.8 & 3.2 \\
\hline University student & 37.3 & 39.9 & 34.4 & 32.3 \\
\hline Graduation from univerisity & 26.7 & 27.4 & 24.1 & 31.2 \\
\hline Graduate student & 12.3 & 9.7 & 15.9 & 11.8 \\
\hline Graduation from graduate school & 3.2 & 4.0 & 1.5 & 5.4 \\
\hline \multicolumn{5}{|l|}{ Annual income } \\
\hline$<1,000,000 \mathrm{JPY}$ & 52.4 & 55.6 & 51.8 & 45.2 \\
\hline $1,000,000-1,999,999$ & 6.3 & 5.6 & 6.7 & 7.5 \\
\hline $2,000,000-2,999,999$ & 9.9 & 12.1 & 7.7 & 8.6 \\
\hline $3,000,000-3,999,999$ & 10.6 & 11.3 & 10.3 & 9.7 \\
\hline $4,000,000-4,999,999$ & 6.7 & 6.0 & 6.2 & 9.7 \\
\hline $5,000,000-5,999,999$ & 4.5 & 3.2 & 5.6 & 5.4 \\
\hline $6,000,000-6,999,999$ & 3.2 & 1.2 & 3.6 & 7.5 \\
\hline $7,000,000-7,999,999$ & 3.0 & 2.0 & 4.6 & 2.2 \\
\hline $8,000,000-8,999,999$ & 0.9 & 0.8 & 1.0 & 1.1 \\
\hline $9,000,000-9,999,999$ & 1.1 & 1.2 & 1.0 & 1.1 \\
\hline$\geqq 10,000,000$ & 1.3 & 0.8 & 1.5 & 2.2 \\
\hline \multicolumn{5}{|l|}{ Marital status } \\
\hline Married & 27.6 & 23.4 & 31.3 & 31.2 \\
\hline Experience of divorce & 1.9 & 1.2 & 1.5 & 4.3 \\
\hline
\end{tabular}

Note: JPY Japanese yen, NPG non-problem gamblers, AG at-risk gamblers, PG pathological gamblers.

\section{Measures}

\section{Demographics}

Participants were asked to answer questions on gender, age, education level, annual income, and marital status.

The South Oaks gambling screen-modified Japanese version (SOGS-J) (Saito 1996)

The South Oaks Gambling Screen (SOGS; Lesieur and Blume 1987) is a 20-item selfreport measure which assesses pathological gambling symptoms. The SOGS produces a 
score ranging from 0 to 20 . A score of 0 indicates that there is no gambling problem ("non-problem gamblers"), 1-4 indicates a possible risk of developing problematic gambling ("at-risk gamblers"), and a score of 5 or more indicates probable pathological gambling ("pathological gamblers"). The Cronbach's alpha in this study was high $(\alpha=0.86)$. In previous studies, individuals that had more pathological gambling symptoms tended to have more erroneous gambling cognitions (Raylu and Oei 2004; Oei et al. 2007). In this study, we expected to find positive moderate associations between the SOGS-J and GRCS-J (the overall score and the factor scores).

\section{Gambling behavior in the previous month}

Participants were asked to indicate the number of days they had gambled in the previous month ("How many days did you gamble in the previous month?") and the amount of money they had spent on gambling ("How much did you spend gambling in the previous month? You do not need to give the income and expenditure on gambling but on money invested"). In this study, on the basis of a previous study (Miller and Currie 2008), we expected to find a significant difference in gambling behavior between individuals who had considerable gambling-related cognitions and those who did not.

\section{Japanese version of the Gambling Related Cognitions Scale (GRCS-J) (Additional file 1)}

The GRCS (Raylu and Oei 2004) assesses five gambling-related cognition domains. The first domain (illusion of control: IC) consists of beliefs that it is possible to control gambling outcomes (e.g., believing that superstitious behavior such as playing the slot machine on the aisle side wall could influence gambling outcomes). The second domain (predictive control: PC) consists of beliefs that it is possible to predict gambling outcomes based on salient cues (e.g., physical conditions or hunches) or past winning or losing data. The third domain (interpretative bias: IB) involves the reframing of gambling outcomes that encourage continued gambling (e.g., attributing winning to one's own skill and losing to one's environment or luck). These first three domains are similar to the gambling-related cognitions identified by Ladouceur and Walker (1996), Toneatto (1999), and Petry (2005). In addition, the GRCS has two other domains, gambling expectancy (GE) and perceived inability to stop gambling (IS), which are related to the development and maintenance of other addictive behaviors (Engels et al. 2005; Young et al. 2006). Similar to substance-use-related cognitions, gambling expectancy refers to beliefs that gambling could result in positive benefits (e.g., gambling makes me happier), and the perceived inability to stop gambling refers to beliefs about the sense of helplessness in controlling gambling urges (Raylu and Oei 2004).

First, a forward translation from the source language (GRCS; Raylu and Oei 2004) into Japanese was independently done by two graduate Japanese students majoring in clinical psychology. Then, an associate professor with a clinical psychological background, who was an English Japanese bilingual and was blind to the original GRCS, translated the provisional GRCS-J back into English. Then, the two English versions of the GRCS (original and back translated) were compared by the first translator and the back-translator and possible discrepancies were discussed until consensus was achieved. The GRCS-J was finalized after four graduate students majoring in clinical psychology and one professor with a clinical psychology background checked the GRCS-J comprehension. 
The GRCS-J is a 23-item questionnaire designed to measure gambling-related cognitions. As with the original GRCS (Raylu and Oei 2004), participants responded using a 7-point Likert scale to indicate the extent to which they agreed with the values expressed in each item. Higher scores indicated a higher number of cognitive distortions.

\section{Procedure}

\section{Questionnaire distribution}

The questionnaires were distributed to the university students in the classroom and were mailed or hand delivered to the workers. A questionnaire package with a consent form and information sheet was distributed to the participants to complete in their own time. We explained that those who did not consent to participation in this study would be placed at no disadvantage whatsoever. The completed questionnaires were returned to the researchers by mail or were put in collection boxes.

\section{Statistical analysis}

Analyses were conducted using IBM SPSS Statistics package 22.0 (SPSS, Inc., Chicago, IL., USA). Descriptive statistics were presented as means and standard deviations $(S D)$ about each variable. A confirmatory factor analysis was used to confirm the factor solutions of the GRCS-J. In the confirmatory factor analysis, the fit indices indicated the extent to which the covariances among the items were accounted for by the hypothesized factor model. We used four fit indices (Chi-square $\left(\chi^{2}\right)$, goodness of fit index (GFI), comparative fit index (CFI), and root mean square error of approximation (RMSEA)), and Cronbach's alphas were also used to assess the internal consistency of the GRCS-J. The concurrent validity of the GRCS-J was measured using the Mann-Whitney test to compare the GRCS-J-high and GRCS-J-low, and Pearson's product-moment correlation coefficients were used between the GRCS-J and the SOGS-J. T-tests were conducted to compare the differences in gamblingrelated cognitions between males and females (Raylu and Oei 2004). We used complete data in this study and did not impute missing values. For all tests, a $p$-value of .05 (two-tailed) was chosen as the statistically significant cutoff.

\section{Ethics}

The study protocol was approved by the Ethics Committee at the Health Sciences University of Hokkaido.

\section{Results}

\section{Demographic characteristics}

Table 1 shows the demographic data and Table 2 shows the descriptive statistics for the participants. The SOGS-J scores identified $46.3 \%(n=248)$ of the participants as "non-problem gamblers", 36.4\% $(n=195)$ as "at-risk gamblers", and $17.4 \%(n=93)$ as "probable pathological gamblers". The percentages for male non-problem gamblers, at-risk gamblers, and pathological gamblers were $51.6 \%(n=128), 73.3 \%(n=143)$, and $86.0 \%(n=80)$, respectively. There were statistically significant differences in relation to the gender percentage among three groups $(\chi 2(2, N=536)=43.78, p<.05)$. However, there were no statistically significant differences with regards to education levels 
Table 2 Age, gambling behaviors, and pathological gambling symptoms

\begin{tabular}{llll}
\hline & NPG & AG & PG \\
& $\mathbf{n}=\mathbf{2 4 8}$ & $\mathbf{n = 1 9 5}$ & $\mathbf{n}=\mathbf{9 3}$ \\
\hline Age & $28.92(11.89)$ & $30.09(11.48)$ & $31.27(12.38)$ \\
Number of days at 1 month & $1.32(0.97)$ & $2.11(3.94)$ & $6.51(6.62)$ \\
Amout of money at 1 month (JPY) & $1853(10631)$ & $10716(27363)$ & $50505(59736)$ \\
SOGS-J & $0.00(0.00)$ & $1.99(1.05)$ & $7.14(2.01)$ \\
\hline
\end{tabular}

Note: JPY Japanese yen, NPG non-problem gamblers, AG at-risk gamblers, PG pathological gamblers, SOGS-J the South Oaks Gambling Screen-Modified Japanese version.

$(\chi 2(14, N=536)=22.34, p=.072)$, annual income $(\chi 2(20, N=536)=20.09, p=.452)$, marital status $(\chi 2(2, N=536)=4.12, p=.127)$, divorce experiences $(\chi 2(2, N=183)=$ $5.41, p=.067)$, or age $(F(2,533)=1.460, p=.233)$ among three groups.

\section{Examination of the GRCS-J factor structure}

Previous studies (Grall-Bronnec et al. 2012; Raylu and Oei 2004; Oei et al. 2007) have identified five factors in the GRCS: IS, IB, IC, GE, and PC. To examine whether the GRCS-J also included these five factors, a confirmatory factor analysis was conducted (Figure 1). The results of the confirmatory factor analysis showed that CFI and RMSEA were in the acceptable range $(\chi 2(229)=931, p<.05$, CFI $=.90$, RMSEA $=.07)$, whereas

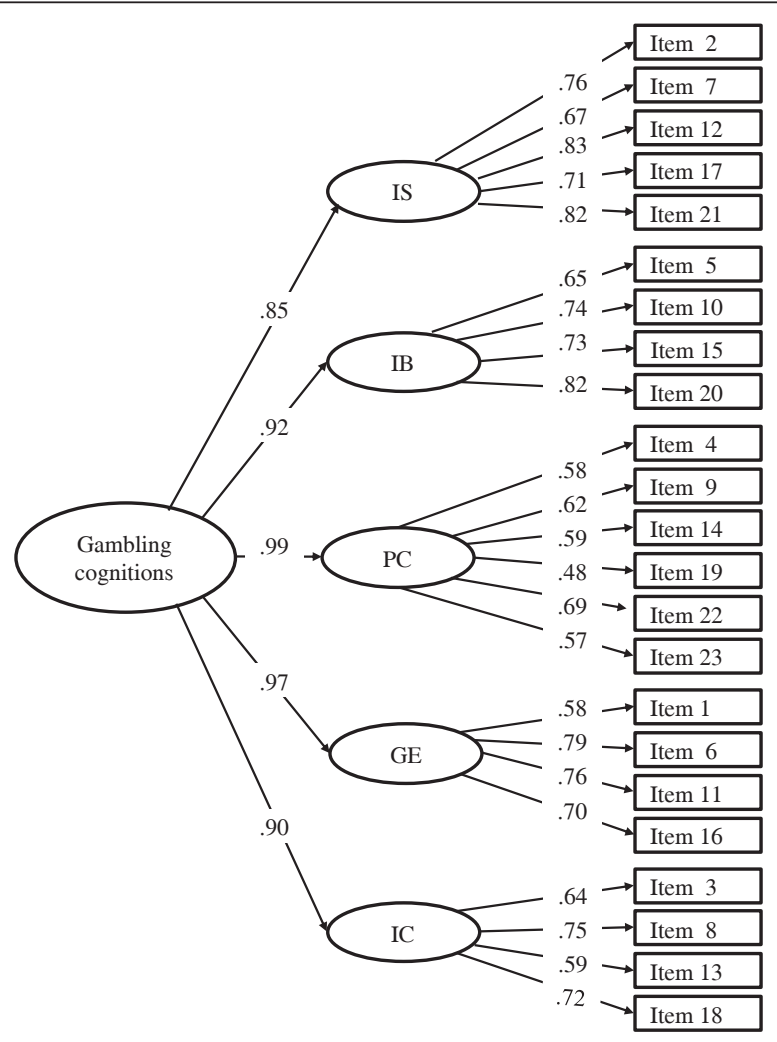

Figure 1 Five-factor model of factorial structure for the GRCS-J. Note: IS perceived inability to stop gambling, IB interpretative bias, GE gambling expectancies, PC predictive control, IC illusion of control. 
GFI was just under 0.9, which was the threshold generally used to interpret the goodness of fit. Furthermore, the level of index about GFI in this study was equivalent to that of the previous study (Grall-Bronnec et al. 2012).

The path coefficients indicating the factor loading for each item were significant and ranged from 0.57 to $0.83(p<.05)$. The correlation coefficients among each GRCS-J factor and between each factor and the total score ranged from 0.53 to 0.89 , suggesting that the factors represented related but distinct constructs (Table 3).

\section{Examination of the GRCS-J reliability and validity}

Reliability

To examine the reliability, we calculated Cronbach's alpha coefficients for the overall scale and for each GRCS-J subscale. Cronbach's alpha coefficients ranged from 0.74 to 0.94, which indicated an acceptable internal consistency (The overall GRCS-J score, $\alpha=.94$; GRCS-J-IS, $\alpha=.85$; GRCS-J-IB, $\alpha=.85$; GRCS-J-PC, $\alpha=.79$; GRCS-J-GE, $\alpha=.76$; GRCS-J-IC, $\alpha=.74$ ).

\section{Validity}

To explore the concurrent validity of the GRCS-J, the Mann-Whitney test and Pearson's product-moment correlation analysis were conducted. First, participants were separated into a "GRCS-high group" ( $n=93$ ) which was those who had scored higher than the average GRCS-J value plus one standard deviation on the total GRCS-J score, and a "GRCS-low group" ( $n=81$ ), which was those who had scored lower than the average GRCS-J value minus one standard deviation on the total GRCS-J score. The differences in the gambling behavior in the previous month between the two groups were then examined. The results showed that the GRCS-high group spent significantly more time and money gambling than the GRCS-low group (the number of days, $U=1359.00, p<.05$ : the amount of money, $U=1387.50, p<.05)$. We then conducted correlation analyses between the GRCS-J (the overall score and the subscales score) and the SOGS-J. As expected, the results of correlation analyses revealed that the total and subscale GRCS-J scores were significantly moderately correlated with the SOGS-J score $(r=.39-.62)$ (Table 3). These results were consistent with those of previous studies (Raylu and Oei, 2004; Oei et al. 2007).

Table 3 Correlation coefficiencies between GRCS-J and SOGS-J

\begin{tabular}{|c|c|c|c|c|c|c|c|}
\hline & GRCS-J & IS & IB & GE & PC & IC & SOGS-J \\
\hline GRCS-J & 1.00 & $.82^{*}$ & $.89^{*}$ & $.89^{*}$ & $.89^{*}$ & $.81^{*}$ & $.61^{*}$ \\
\hline GRCS-J-IS & & 1.00 & $.67^{*}$ & $.69^{*}$ & $.58^{*}$ & $.53^{*}$ & $.62^{*}$ \\
\hline GRCS-J-IB & & & 1.00 & $.76^{*}$ & $.76^{*}$ & $.64^{*}$ & $.55^{*}$ \\
\hline GRCS-J-GE & & & & 1.00 & $.73^{*}$ & $.65^{*}$ & $.57^{*}$ \\
\hline GRCS-J-PC & & & & & 1.00 & $.73^{*}$ & $.46^{*}$ \\
\hline GRCS-J-IC & & & & & & 1.00 & $.39^{*}$ \\
\hline SOGS-J & & & & & & & 1.00 \\
\hline
\end{tabular}

Note: GRCS-J the Japanese version of the Gambling Related Cognitions Scale, SOGS-J The South Oaks Gambling Screen-Modified Japanese version, IS perceived inability to stop gambling, IB interpretative bias, GE gambling expectancies, PC predictive control, IC illusion of control, $n=536 ;{ }^{*} p<.05$. 
Examination of the gender difference about gambling-related cognitions in the Japanese sample

To examine gender differences in the subscale and total GRCS-J scores, we conducted $t$-tests (Table 4). The results revealed that there were statistically significant gender differences (GRCS-J: $t(465.828)=6.02, p<.05$, GRCS-J-IS: $t(504.091)=5.47, p<.05$, GRCS-J-IB: $t(447.751)=5.10, p<.05$, GRCS-J-GE: $t(453.569)=6.23, p<.05$, GRCS-J-PC: $t(534)=4.69, p<.05$, GRCS-J-IC: $t(431.155)=3.69, p<.05)$. The results showed that males had more irrational gambling cognitions than females.

\section{Discussion}

The purpose of this study was to develop a GRCS-J which could accurately assess gambling-related cognitions in a Japanese community sample, and to examine its reliability and validity. The results demonstrated that the GRCS-J was a valid and reliable measure for assessing gambling-related cognitions in Japanese. From these results, researchers and clinicians could confidently use the GRCS-J to assess gambling-related cognitions in Japanese speaking people. We also conducted study on gender differences as one of the characteristics of the gambling-related cognitions in Japanese people, and found that the males in the sample had more erroneous gambling cognitions than the females.

A previous study had determined that there was a five-factor model in the GRCS; perceived inability to stop gambling, interpretative bias, illusion of control, gambling expectancies, and predictive control (Grall-Bronnec et al. 2012; Raylu and Oei 2004; Oei et al. 2007). Similar to the GRCS (Raylu and Oei 2004) and the GRCS-C (Oei et al. 2007), our study confirmed that the GRCS-J was also composed of a five-factor model. All five GRCS-J factors were moderately to highly correlated with each factor and with the total GRCS-J score. Cronbach's alpha coefficients for the total and subscale GRCS-J scores were similar to those found in the original GRCS (Raylu and Oei 2004), indicating that the GRCS-J also had acceptable internal consistency.

We also verified the concurrent validity of the GRCS-J, and indicated that there were significant gambling behavior differences (the number of days and the amount of money) in the previous month between the GRCS-high group and the GRCS-low group. These results were consistent with the results of previous studies which had reported that gamblers with greater gambling-related cognitions were likely to gamble more frequently and spend higher amounts on gambling (Miller and Currie 2008). In addition, similar to a previous study (Grall-Bronnec et al. 2012; Raylu and Oei 2004;

Table 4 Gender differences of the GRCS-J

\begin{tabular}{lllll}
\hline & $\begin{array}{l}\text { Male } \\
(\mathbf{n}=\mathbf{3 5 1})\end{array}$ & $\begin{array}{l}\text { Female } \\
(\mathbf{n}=\mathbf{1 8 5})\end{array}$ & $\mathbf{t}$ value & $\mathbf{d}(\mathbf{9 5 \%} \mathbf{C l})$ \\
\hline GRCS-J & $57.32(26.39)$ & $45.00(20.19)$ & $6.02^{*}$ & $0.50(0.32$ to 0.69$)$ \\
GRCS-J-IS & $8.49(4.81)$ & $7.04(4.07)$ & $3.69^{*}$ & 0.31 (0.14 to 0.50) \\
GRCS-J-IB & $10.72(6.31)$ & $8.16(5.09)$ & $5.10^{*}$ & $0.43(0.25$ to 0.61) \\
GRCS-J-PC & $15.92(7.06)$ & $13.04(6.18)$ & $4.88^{*}$ & $0.43(0.25$ to 0.61) \\
GRCS-J-GE & $10.54(5.19)$ & $7.98(4.12)$ & $6.23^{*}$ & 0.53 (0.35 to 0.71) \\
GRCS-J-IC & $11.65(7.21)$ & $8.78(4.83)$ & $5.47^{*}$ & 0.44 (0.26 to 0.62) \\
\hline
\end{tabular}

Note: GRCS-J the Japanese version of the Gambling Related Cognitions Scale, Cl confidence interval, IS perceived inability to stop gambling, IB interpretative bias, GE gambling expectancies, PC predictive control, IC illusion of control, ${ }^{*} \mathrm{p}<.05$. 
Oei et al. 2007), the results of correlation analyses found that increased gamblingrelated cognitions were related to a higher level of pathological gambling symptoms. These results were consistent with previous research in which it was found that probable pathological gamblers were more likely to make erroneous predictions on gambling outcomes, had more positive gambling expectancies, and increased cognitive dysfunctions in their inability to stop gambling (Oei et al. 2007; Gillespie et al. 2007).

In addition, significant gender differences were found in the GRCS-J subscale scores and the total score. Consistent with other studies (Grall-Bronnec et al. 2012; Raylu and Oei 2004; Oei et al. 2007), males had more irrational gambling-related cognitions than females in our study. In a previous study, Goodie and Fortune (2013) indicated that gambling-related cognitions were significantly related to gambling severity (Goodie and Fortune 2013). Gillespie et al. (2007) indicated that gamblers who had more irrational gambling cognitions were apt to gamble continually. Given these indications, we expect that males would have higher rates of pathological gambling than females in Japan.

In this study, $17.4 \%(n=93)$ of the participants were classified as probable pathological gamblers. This rate is much higher than other epidemiological studies on pathological gambling (Shaffer et al. 1999; Volberg and Abbott 1994; Wong and So 2003). One of the main reasons for this high rate of pathological gamblers in Japan is the fact that in this study there were a higher proportion of participants either in their late teens or early twenties. American Psychiatric Association (2000) reported that the prevalence of pathological gamblers is approximately $0.4-3.4 \%$ in adults, and the prevalence at an earlier age is about $2.8-8.0 \%$. Sixty-four percent of the participants in our study were either in their late teens or early twenties, which might cause the high rates of pathological gamblers. Moreover, the proportion of male participants was 65.5\%. Given the relationship between the sex and pathological gambling (Johansson et al. 2009), this high percentage of male participants might have the impact on the level of pathological gamblers prevalence in the sample. We also used the SOGS-J (Saito 1996) to assess for pathological gamblers and, although the SOGS (Lesieur and Blume, 1987) demonstrated good classification accuracy in the gambling treatment sample, it has had poorer accuracy in general population samples with a $50 \%$ false positive rate (Stinchfield 2002). Tang and colleagues proposed a SOGS cut score of 8 to screen for probable pathological gambling in Chinese people (Tang et al. 2010). Because we used a SOGS cut score of 5 in accordance with a previous study (Lesieur and Blume 1987), the prevalence rate for pathological gamblers in this study might be overestimated. In this study, when we used a SOGS cut score of 8 , the prevalence of probable pathological gamblers was $6.7 \%(n=36)$. In the future, the classification accuracy of SOGS should be examined further in Japan.

\section{Limitations}

One potential limitation of this study was that the sample was a community sample. Although the psychometric properties of the GRCS-J were promising, it did not indicate whether the results could be generalized outside a community group, such as in a clinical sample. Given that the GRCS-J would be used in the clinical assessment and treatment of pathological gambling, it is necessary to assess the psychometric properties of the GRCS-J and to replicate the factor structure using a clinical sample. 
Another limitation of this present study is the reliability and the stability of the GRCS-J. In clinical settings, it is recommended that follow-up assessments would be conducted at four time-points (post-treatment, short-term, medium-term, and long-term follow-up) (Walker et al. 2006). Future studies need to examine the test-retest reliability of the GRCS-J for use in clinical settings.

\section{Conclusions}

The GRCS-J has been shown to have good psychometric properties in this study. Therefore, it is indicated that the GRCS-J was a valid and reliable measure for assessing gambling cognitions among a non-clinical Japanese sample. This would help researchers and clinicians to identify the types of gambling-related cognitions among Japanese gamblers and to understand the role of these cognitions in the development and maintenance of pathological gambling.

\section{Additional file}

Additional file 1: The Japanese version of the GRCS.

Competing interests

The authors declare that they have no competing interests.

Authors' contributions

KY and TT carried out data collection and drafted the article. JK and YS revised and contributed to article contents. All authors read and approved the final manuscript.

\section{Acknowledgment}

The authors would like to thank Enago (www.enago.jp) for the English language review and Mr. Kyohei Tadano for helping me to translate English into Japanese.

\section{Author details}

${ }^{1}$ Tobacco Academic Studies Center, Tokyo, Japan. ${ }^{2}$ Faculty of Education and Culture, University of Miyazaki, Miyazaki, Japan. ${ }^{3}$ School of Psychological Science, Health Sciences University of Hokkaido, Sapporo, Japan.

Received: 16 November 2014 Accepted: 5 February 2015

Published online: 25 February 2015

\section{References}

American Psychiatric Association. (2000). Diagnostic and statistical manual of mental disorder (4th ed.). Washington, D. C: American Psychiatric Association. TR.

Blaszczynski, A, \& Nower, L. (2002). A pathways model of problem and pathological gambling. Addiction, 97, 487-499.

Cowlishaw, S., Merkouris, S., Dowling, N., Anderson, C., Jackson, A., \& Thomas, S. (2012) Psychological therapies for pathological and problem gambling. Cochrane Database of Systematic Reviews, The Cochran Library, DOI: 10.1002/ 14651858.CD008937.pub2.

Engels, RCME, Wiers, R, Lemmers, L, \& Overbeek, G. (2005). Drinking motives, alcohol expectancies, self-efficacy, and drinking patterns. Journal of Drug Education, 35, 147-166.

Fortune, EE, \& Goodie, SA. (2012). Cognitive distortions as a component and treatment focus of pathological gambling: A review. Psychology of Addictive Behaviors, 26, 298-310.

Gaboury, A, \& Ladouceur, R. (1989). Erroneous perceptions and gambling. Journal of Social Behavior and Personality, 4(4), 411-420.

Gillespie, MAM, Derevensky, J, \& Gupta, R. (2007). The utility of outcome expectancies in the prediction of adolescent gambling behaviour. Journal of Gambling Issues, 19, 69-86.

Goodie, SA, \& Fortune, EE. (2013). Measuring cognitive distortions in pathological gambling: Review and meta-analyses. Psychology of Addictive Behavior, 27, 730-743.

Grall-Bronnec, M, Bouju, G, Sébille-Rivain, V, Gorwood, P, Boutin, C, Vénisse, J, \& Hardouin, J. (2012). A French adaptation of the gambling-related cognitions scale (GRCS): A useful tool for assessment of irrational thoughts among gamblers. Journal of Gambling Issues, 27, 1-21.

Johansson, A, Grant, EJ, Kim, WS, Odlaug, LB, \& Götestam, GK. (2009). Risk factors for problematic gambling: A critical literature review. Journal of Gambling Studies, 25, 67-92.

Ladouceur, R, Sylvain, C, Boutin, C, Lachance, S, Doucet, C, Leblond, J, \& Jacques, C. (2001). Cognitive treatment of pathological gambling. The Journal of Nervous and Mental Disease, 189(11), 774-780.

Ladouceur, R, \& Walker, M. (1996). A cognitive perspective on gambling. In PM Salkovskis (Ed.), Trends in cognitive and behavioural therapies (pp. 89-120). New York: Wiley. 
Lesieur, HR, \& Blume, SB. (1987). The South Oaks Gambling Screen (SOGS): A new instrument for the identification of pathological gamblers. American Journal of Psychiatry, 144(9), 1184-1188.

Lorains, KF, Cowlishaw, S, \& Thomas, AS. (2011). Prevalence of comorbid disorders in problem and pathological gambling: Systematic review and meta-analysis of population survey. Addiction, 106, 490-498.

Miller, NV, \& Currie, SR. (2008). A Canadian population level analysis of the roles of irrational gambling cognitions and risky gambling practices as correlates of gambling intensity and pathological gambling. Journal of Gambling Studies, 24(3), 257-274

Moriyama, N. (2008). Clinical features of 100 pathological gamblers. Clinical Psychiatry, 50(9), 895-904.

Oei, TPS, Lin, J, \& Raylu, N. (2007). Validation of the Chinese version of the Gambling Related Cognitions Scale (GRCS-C) Journal of Gambling Studies, 23(3), 309-322.

Petry, MN. (2005). Pathological Gambling; Etiology, Comorbidity, and Treatment. Washington, DC: American Psychologica Association.

Raylu, N, \& Oei, TPS. (2004). The Gambling Related Cognitions Scale (GRCS): Development, confirmatory factor validation and psychometric properties. Addiction, 99(6), 757-769.

Sato, T. (2008). So-called gambling addiction. Human mind, 139, 36-40.

Saito, S. (1996). Compulsive/pathological gambling and its treatment; A introduction of a gambling screen (SOGS-Modified Japanese Version). Alcohol Dependence \& Addiction, 13(2), 102-109.

Shaffer, JH, Hall, NM, \& Vander Bilt, J. (1999). Estimating the prevalence of disordered gambling behavior in the United States and Canada: A research synthesis. American Journal of Public Health, 89(9), 1369-1376.

Stinchfield, R. (2002). Reliability, validity and classification accuracy of the South Oaks Gambling Screen (SOGS). Addictive Behavior, 27, 1-19.

Stucki, S, \& Rihs-Middel, M. (2007). Prevalence of adult problem and pathological gambling between 2000 and 2005: An update. Journal of Gambling Studies, 23, 245-257.

Tang, SC, Wu, SMA, Tang, CYJ, \& Yan, WCE. (2010). Reliability, validity, and cut scores of the South Oaks Gambling Screen (SOGS) for Chinese. Journal of Gambling Studies, 26, 145-158.

Toneatto, T. (1999). Cognitive psychopathology of problem gambling. Substance Use \& Misuse, 34(11), 1593-1604.

Toneatto, T, Blitz-Miller, T, Calderwood, K, Dragonetti, R, \& Tsanos, A. (1997). Cognitive distortions in heavy gambling. Journal of Gambling Studies, 13(3), 253-266.

Toneatto, T, \& Ladouceur, R. (2003). Treatment of pathological gambling: A critical review of the Literature. Psychology of Addictive Behaviors, 17(4), 284-292

Volberg, AR, \& Abbott, MW. (1994). Lifetime prevalence estimates of pathological gambling in New Zealand. International Journal of Epidemiology, 23(5), 976-983.

Walker, M, Toneatto, T, Potenza, NM, Petry, MN, Ladouceur, R, Hodgins, CD, el-Guebaly, N, Echeburua, E, \& Blaszczynski, A. (2006). A framework for reporting outcomes in problem gambling treatment research: the Banff, Alberta Consensus. Addiction, 101(4), 504-511.

Whelan, PJ, Steenbergh, AT, \& Meyers, WA. (2007). Problem and pathological gambling. Washington: Hogrefe \& Huber Publishers.

Wong, ILK, \& So, EMT. (2003). Prevalence estimates of problem and pathological gambling in Hong Kong. American Journal of Psychiatry, 160(7), 1353-1354.

Young, RM, Connor, JP, Ricciardelli, LA, \& Saunders, JB. (2006). The role of alcohol expectancy and drinking refusal self-efficacy beliefs in university student drinking. Alcohol \& Alcoholism, 41(1), 70-75.

Submit your manuscript to a SpringerOpen ${ }^{\oplus}$ journal and benefit from:

- Convenient online submission

Rigorous peer review

- Immediate publication on acceptance

Open access: articles freely available online

- High visibility within the field

- Retaining the copyright to your article

Submit your next manuscript at $>$ springeropen.com 